\title{
Erratum: New Lower Bounds on the Self-Avoiding-Walk Connective Constant ${ }^{1}$
}

\author{
Takashi Hara, ${ }^{2}$ Gordon Slade, $^{3}$ and Alan D. Sokal ${ }^{4}$
}

Received August 30, 1994

Due to a coding error in our calculation of the integral

$$
C_{0}\left(0, x ; \frac{1}{2 d}\right)=\int_{[-\pi, \pi]^{d}} \frac{d^{d} k}{(2 \pi)^{d}} \frac{e^{i k \cdot x}}{1-d^{-1} \sum_{j=1}^{d} \cos k_{j}}
$$

the values given in the second column of Table IV contain errors in their final four digits. The correct values are given here in Table I. The error is negligible $\left(<10^{-17}\right)$ for $d \geqslant 4$. These errors do not affect other numerical values quoted, apart from a change in the final digit of the $d=3,(0,0)$ entry of Table II, whose correct value is slightly improved to 3.956776 .

The integral $C_{0}(0,0 ; 1 /(2 d))$ is equal to the expected number of returns to the origin of the simple random walk in $\mathbb{Z}^{d}$. For $d=3$, this has been computed exactly in terms of an elliptic integral in ref. 1 and in terms of the gamma function in ref. 2. The latter contains an erroneous numerical prefactor, which when corrected as indicated in ref. 3 yields the exact expression

$$
C_{0}\left(0,0 ; \frac{1}{6}\right)=\frac{\sqrt{6}}{32 \pi^{3}} \Gamma\left(\frac{1}{24}\right) \Gamma\left(\frac{5}{24}\right) \Gamma\left(\frac{7}{24}\right) \Gamma\left(\frac{11}{24}\right) \quad(d=3)
$$

\footnotetext{
${ }^{1}$ This paper appeared in J. Stat. Phys. 72:479-517 (1993).

2 Department of Applied Physics, Tokyo Institute of Technology, Oh-Okayama, Meguro-ku, Tokyo 152, Japan. E-mail: hara@appana.ap.titech.ac.jp.

${ }^{3}$ Department of Mathematics and Statistics, McMaster University, Hamilton, Ontario, Canada L8S 4K1. E-mail: slade ầmcmaster.ca.

4 Department of Physics, New York University, New York, New York 10003. E-mail: sokal@ac[4.nyu.edu.
} 
Table I. Corrected Values of $C_{0}(0, x ; 1 /(2 d))$ for $d=3$, Rounded to Ten Digits after the Decimal

\begin{tabular}{cc}
\hline$x$ & $C_{0}(0, x ; 1 /(2 d))$ for $d=3$ \\
\hline$(0,0,0)$ & 1.5163860592 \\
$(1,0,0)$ & 0.5163860592 \\
$(1,1,0)$ & 0.3311486021 \\
$(1,1,1)$ & 0.2614701264 \\
$(2,0,0)$ & 0.2573358873 \\
$(2,1,0)$ & 0.2155896208 \\
$(2,1,1)$ & 0.1917916506 \\
$(2,2,0)$ & 0.1683310356 \\
$(2,2,1)$ & 0.1569524128 \\
$(3,0,0)$ & 0.1652707810 \\
$(3,1,0)$ & 0.1531388988 \\
$(3,1,1)$ & 0.1441957103 \\
$(3,2,0)$ & 0.1324510731 \\
$(4,0,0)$ & 0.1217332037 \\
$(4,1,0)$ & 0.1171304972 \\
$(5,0,0)$ & 0.0966064520 \\
\hline
\end{tabular}

Evaluation of the right side using both Mathematica and Maple gives $1.51638605915197801815 \ldots$, which agrees with our entry here in Table I and with the value 1.516386059 from ref. 1 but disagrees with the final digits of the value $1.516386059137 \ldots$ quoted on p. 126 of ref. 3.

Finally, in an unrelated matter, there is a typographical error in the $d=3,(2,2)_{\text {opt }}$ entry of Table II, whose correct value is 4.476141 .

\section{REFERENCES}

1. G. N. Watson, Three triple integrals, Q. J. Math. (Oxford) 10:266-276 (1939).

2. M. L. Glasser and I. J. Zucker, Extended Watson integrals for the cubic lattices, Proc. Natl. Acad. Sci. USA 74:1800-1801 (1977).

3. P. G. Doyle and J. L. Snell, Random Walks and Electric Networks (Mathematical Association of America, 1984). 